\title{
CHARACTERIZATION OF ANTIGEN SAMPLING CELLS IN RAINBOW TROUT GILL EPITHELIUM
}

\author{
Goshi Kato $^{1 \S}$, Haruya Miyazawa ${ }^{1}$, Takuya Yamaguchi ${ }^{2}$, Hidehiro Kondo ${ }^{1}$, Motohiko Sano ${ }^{1}$, \\ Uwe Fischer ${ }^{2}$ \\ ${ }^{1}$ Tokyo University of Marine Science and Technology, Konan 4-5-7, Minato-ku, Tokyo, 108- \\ 8477, Japan \\ ${ }^{2}$ Friedrich-Loeffler-Institut, Südufer 10, Greifswald-Insel Riems, 17493, Germany
}

\begin{abstract}
In mammalian mucosal surfaces, microfold cells (M cells) take up antigens and deliver it to antigen presenting cells in lymphoid tissues such as Payer's patches. In rainbow trout, a part of gill epithelial cells take up Aeromonas salmonicida subsp. salmonicida (A.s.s.) bacterin during bath-vaccination. Antigen-sampling cells in gill epithelium could be stained with lectin Ulex europaeus agglutinin 1 (UEA-1) but not with wheat germ agglutinin (WGA); this staining pattern is characteristic for mammalian $\mathrm{M}$ cells. In this study, we sorted A.s.s. ${ }^{+}$UEA- ${ }^{+}$cells from rainbow trout gill epithelium and performed a comprehensive gene expression analysis using next generation sequencing to characterize the corresponding antigen sampling cells.

Rainbow trout (approx. $150 \mathrm{~g}$ ) were bath-vaccinated with Syto61-stained A.s.s. bacterin. Epithelial cells were isolated from the gills and stained with FITC-labeled UEA-1 and subjected to flow cytometry followed by sorting. Two cell populations, A.s.s. ${ }^{+}$UEA-1 ${ }^{+}$and A.s.s. ${ }^{+}$UEA-1 ${ }^{-}$ were isolated and total RNA was extracted from each cell population. The cDNA library of each cell population was sequenced using a MiSeq system and gene expression analysis based on the read counts was performed using Trinity assemble software.

Flow cytometry revealed that there were two distinct cell populations containing the A.s.s. bacterin in the epithelium: A.s.s. ${ }^{+}$UEA- $1^{+}$and A.s.s. ${ }^{+}$UEA-1 ${ }^{-}$. The percentage of each cell group was $22 \%$ in A.s.s. ${ }^{+}$UEA- $1^{+}$, and $5 \%$ in A.s.s. ${ }^{+}$UEA-1 ${ }^{-}$population. These two populations also showed different scatter characteristics in flow cytometry, where the A.s.s. ${ }^{+}$UEA- $^{+}$ population exhibited a relatively high side scatter as a measure for pronounced granulation. Remarkably, the A.s.s. ${ }^{+}$UEA $-1^{+}$population highly expressed annexin which is typical for mammalian M cells, and interleukin (IL)-20 which is typical for mammalian epithelial cells. In contrast, A.s.s. $^{+}$UEA-1 $^{-}$cells showed high expression levels of CD83 and IL-12 that are typical for mammalian macrophages and dendritic cells. MHC class II related genes were highly expressed in both of the cell populations from rainbow trout gills. These results suggest that almost of the bacterin are taken up by the UEA- $1^{+}$antigen-sampling cells which show properties of epithelial cells during bath-vaccination. In addition, macrophage/dendritic cell lineage may also take part in the uptake of the bacterin.
\end{abstract}

\section{KEYWORDS}

antigen up take, antigen sampling cell, mucosal immunity, rainbow trout, next generation sequencer

$\S$ Corresponding author. Tel: +81-3-5463-0462

E-mail address: gkato00@kaiyodai.ac.jp 